\title{
Aplicación de Vacío en la Deshidratación Osmótica de Higos (ficus carica)
}

\author{
Sandra I. Arreola y Martha E. Rosas \\ Facultad de Estudios Superiores Cuautitlán, UNAM, Av. 1॰ de mayo s/n Colonia Atlanta, \\ CP 4740 Cuautitlán Izcalli, Estado de México-México
}

\begin{abstract}
Resumen
Se determinan los coeficientes de difusión efectivos y los coeficientes de transferencia de masa para la humedad perdida y para los sólidos ganados en el proceso de deshidratación osmótica de higos (ficus carica), a tres concentraciones de sacarosa en solución (55, 65 y $75 \%)$ y dos temperaturas (35 y $45^{\circ} \mathrm{C}$ ), bajo vacío constante (aprox. $40 \mathrm{kPa}$ ). Se consideró una geometría esférica y estado inestable, así como una cinética de primer orden. Los coeficientes de difusión y de transferencia de materia para agua incrementaron con la temperatura y los valores mayores se obtuvieron para una concentración de $65 \%$ de azúcar y $35^{\circ} \mathrm{C}$. La concentración de la solución osmótica y la temperatura influyen considerablemente durante el proceso debido a que un aumento de esta última intensifica la eliminación de agua y la penetración de sacarosa. Además, el vacío contribuye a la apertura de la estructura del higo, disminuyendo el tiempo de proceso.
\end{abstract}

Palabras clave: transferencia de materia, deshidratación osmótica, ficus carica, higos

\section{Vacuum Application in Osmotic Dehydration of Figs (ficus carica)}

\begin{abstract}
The aim of this work was the effective diffusion coefficients, water loss and solid gain mass transfer coefficients calculation ( $\mathrm{De}_{\mathrm{w}}, \mathrm{De}_{\mathrm{s}}, \mathrm{K}_{\mathrm{w}} \mathrm{y} \mathrm{K}_{\mathrm{s}}$ respectively) from an osmotic dehydration of figs, at three different sucrose solution concentration $(55,65$ and $75 \%)$ and two temperatures ( 35 and $\left.45^{\circ} \mathrm{C}\right)$, under constant vacuum (39996.6 Pa); a spherical geometry and unsteady state was consider as well as a firs order kinetic. As a rule, the water diffusion and mass transfer coefficients increased with temperature, and the main values were obtained for a $65 \%$ sucrose concentration and $35^{\circ} \mathrm{C}$. In this way we show the variables mentioned effect in the mass transfer and time process, which represents advantages on other dehydration operations. The osmotic solution concentration and temperature affects during process considerably owing to a temperature increase that intensify water elimination and sucrose penetration, in addition to the vacuum pressure that contribute to open the fig structure, decreasing the time process.
\end{abstract}

Keywords: mass transfer, osmotic dehydration, ficus carica, figs 


\section{INTRODUCCIÓN}

El proceso de deshidratación osmótica es frecuentemente aplicado para conservar la calidad y estabilidad de frutas y hortalizas, sin tener pérdidas considerables en compuestos aromáticos; además de que puede ser utilizado como una operación previa en el secado y la liofilización, reduciéndose así los costos energéticos. La deshidratación osmótica de alimentos incluye dos tipos de transferencia de masa: la difusión del agua del alimento a la solución y la difusión de solutos de la solución al alimento. En el primer tipo, la fuerza conductora de la transferencia de masa es la diferencia de presión osmótica, mientras en la segunda es la diferencia de concentraciones (Barbosa-Canovas, 2000). Es un tratamiento de eliminación parcial de agua, donde se sumerge la materia prima en una solución hipertónica que tiene una alta presión osmótica y baja actividad de agua, siendo la fuerza impulsora para que el agua del alimento se difunda en el medio, originándose así una transferencia de masa desde la región de mayor concentración hacia la de menor concentración (Kaymak y Sultanoglu, 2000).

En los productos deshidratados osmóticamente la mayor resistencia a la transferencia de masa se localiza en la membrana celular semipermeable la cual depende de las características y morfología de los productos; y a través de la cual es posible la transferencia de agua, sales, y azucares naturales (glucosa y fructosa). Las variables de proceso, que son: la concentración de la solución osmótica, el tipo de soluto, la temperatura, la presión, los tiempos de residencia, la geometría y el tipo de tejido, han sido estudiadas extensamente, ya que todas tienen una considerable influencia en la velocidad de la transferencia de masa. El aumento de la temperatura en el sistema va a producir cambios en la permeabilidad de la membrana celular y en la fluidez de la solución osmótica. Cuando se aplica una presión de vacío se favorece el proceso de transferencia de masa ya que permite retirar los gases ocluidos en espacios intracelulares, característicos del tejido parenquimatoso, y ser ocupados por la solución osmótica, incrementando el área disponible para a transferencia de masa. La concentración de la solución afecta a la cinética de la deshidratación, ya que si se mantiene el gradiente de concentraciones, se favorece la velocidad de transferencia (Rastogi et al., 2002).
El vacío acelera el intercambio del soluto hacia la matriz gracias a una forzada y pronta penetración de la solución; esto es mayormente favorable para la extracción del agua, como las moléculas del agua pueden migrar más fácilmente en los poros intercelulares llenados con líquido, llevando niveles de pérdida de agua más altos. (Jongen Wim, 2002)

La transferencia de masa toma lugar en las primeras dos horas de el proceso para conseguir la perdida de agua y en los primeros treinta minutos la ganancia de sólidos puede ocurrir (Gomes et al., 2005).

Los agentes osmóticos más utilizados son soluciones hipertónicas de azúcar (sacarosa) y sal (coluro de sodio), (Chenlo et al., 2006). Debido a que es muy importante para el proceso el tipo de soluto a utilizar, se eligió emplear sacarosa debido a que por su eficacia, conveniencia y sabor agradable se ha encontrado que es uno de los mejores agentes osmóticos, además de que se ocupa para frutas principalmente; y también es un inhibidor eficaz de la polifenoloxidasa, evita la perdida de sabores volátiles y la mayoría de las membranas celulares son permeables a ella (Sharma ,2003).

Las frutas tropicales en su conjunto, se encuentran en el quinto puesto de importancia mundial, con una producción total de 46,3 millones de toneladas. El higo se considera una fruta tropical común y es procedente de la cosecha de la higuera. Normalmente es de tamaño pequeño, de 6.0 a $7.0 \mathrm{~cm}$ de largo y de 4.5 a $5.5 \mathrm{~cm}$ de diámetro, se presenta en diferentes formas, según la variedad a la que pertenezca: ovalado u oviforme o también achatado en la base y más ancho que alto en muchas ocasiones. Su piel, según la especie es verde pálido, negra o morada; comestible, pero por lo general, no se consume; consistente y tersa cuando no esta maduro y mostrando arrugas y aberturas en el momento en el que el fruto se encuentra en perfecto estado de consumo. La pulpa, blanquecina, rosa pálido o morada esconde cientos de granos diminutos que no estorban al ser comido el fruto. Los higos frescos son una mercancía sumamente delicada y perecedera; son muy ricos en azucares, proteínas, fibras, minerales sobre todo calcio, fósforo y hierro (Sánchez, 2004). 


\section{MATERIALES Y MÉTODOS}

Los higos fueron adquiridos en un supermercado local, provenientes del estado de Veracruz, en un estado de madurez comercial, y fueron seleccionados con un diámetro aproximado de $5 \mathrm{~cm}$. La humedad inicial se determinó por el método 934.06 del AOAC (1990), siendo del $86.8 \%$ en base húmeda. El proceso de deshidratación osmótica se llevo a cabo en una cámara cilíndrica de vidrio diseñada para este propósito, aplicando un vacío constante, colocada sobre una parilla eléctrica para mantener la temperatura.

Se prepararon $1850 \mathrm{ml}$ de solución osmótica de sacarosa para cada concentración $(55,65$ y $75 \%$ ) y se introdujeron $100 \mathrm{~g}$ de muestra aproximadamente. Para cada combinación de temperatura $\left(35\right.$ y $\left.45^{\circ} \mathrm{C}\right)$ y concentración de la solución osmótica; los higos se pesaron cada 10 minutos, hasta el término del tiempo de proceso que fue de $100 \mathrm{~min}$, en una balanza digital OHAUS modelo As200, retirándoles previamente el exceso de solución osmótica con una toalla absorbente. La humedad se les determinó por el método 934.06 del AOAC (1990); la concentración en grados Brix se verificó por medio de un refractómetro digital Leica modelo AR200.

Los coeficientes de difusión efectiva se obtuvieron de la solución analítica a la segunda ley de Fick, para la geometría de esfera y en estado inestable, considerando difusión constate en el proceso, humedad inicial uniforme y difusión radial (Crank, 1975):

$$
\begin{aligned}
& M R=\frac{M_{t}-M_{\infty}}{M_{0}-M_{\infty}}=\frac{6}{\pi^{2}} \sum_{n=1}^{\infty} \frac{1}{n^{2}} \exp \left(\frac{-D e_{w} n^{2} \pi^{2} t}{r^{2}}\right) \\
& S R=\frac{S_{t}-S_{\infty}}{S_{0}-S_{\infty}}=\frac{6}{\pi^{2}} \sum_{n=1}^{\infty} \frac{1}{n^{2}} \exp \left(\frac{-D e_{s} n^{2} \pi^{2} t}{r^{2}}\right)
\end{aligned}
$$

donde: MR y SR son las proporciones de humedad y de solutos, respectivamente; los subíndices $0, \infty$ y $\mathrm{t}$ representan las concentraciones iniciales, en equilibrio y a cualquier tiempo; $\mathrm{De}_{\mathrm{w}}$ y $\mathrm{De}_{\mathrm{s}}$ son los coeficientes de difusión efectiva $\left(\mathrm{m}^{2} / \mathrm{s}\right)$ de agua y solutos respectivamente. Para tiempos largos, las ecuaciones 1 y 2 fueron ajustadas para el cálculo de la difusión efectiva:

$$
M R=\frac{M t-M \infty}{M o-M \infty}=\frac{6}{\pi^{2}} \exp -\pi^{2} \frac{D e_{w} t}{r^{2}}
$$

$S R=\frac{S t-S_{\infty}}{S_{0}-S_{\infty}}=\frac{6}{\pi^{2}} \exp -\pi^{2} \frac{D e_{s} t}{r^{2}}$

Para la transferencia de masa aproximada al equilibrio, se considero una cinética de primer orden, donde los coeficientes fueron calculados de la siguiente manera (Rastogi y Raghavarao, 2002 y 2004; Pan et al.,2003):

$$
\begin{aligned}
& -\frac{d m}{d t}=K_{w}\left(M_{t}-M_{\infty}\right) \\
& \frac{d s}{d t}=K_{s}\left(S_{t}-S_{\infty}\right)
\end{aligned}
$$

El diseño experimental fue un factorial $2 \times 3$, con tres réplicas en cada tratamiento, obteniendo como el factor de mayor influencia, tanto en la pérdida de humedad como en la ganancia de sólidos, a la temperatura.

Cada experimento se llevó a cabo por triplicado, los comportamientos promedio fueron ajustados estadísticamente por intervalos de confianza al $95 \%$.

\section{RESULTADOS Y DISCUSIÓN}

La variación en el contenido de humedad y de sólidos con respecto al tiempo de inmersión se presenta en las figuras 1 y 2 , donde se observa que la velocidad de pérdida de agua se incrementa conforme se incrementa la concentración de la solución osmótica y la temperatura. De la variación en el contenido de humedad y sólidos con respecto al tiempo, se obtuvo la velocidad de cambio del contenido de humedad $(-\mathrm{dm} / \mathrm{dt})$ y del contenido de sólidos (ds/dt) en función de los contenidos promedio de humedad y sólidos, respectivamente, para inferir los contenidos en equilibrio $\left(\mathrm{M}_{\infty}, \mathrm{S}_{\infty}\right)$, así como para obtener los coeficientes de transferencia de masa $\left(\mathrm{K}_{\mathrm{w}}, \mathrm{K}_{\mathrm{s}}\right)$ que se muestran en la tabla 1.

Tabla 1: Coeficiente de transferencia de masa para humedad y sólidos en higos

\begin{tabular}{|c|c|c|c|c|c|}
\hline$\%$ & ${ }^{\circ} \mathrm{C}$ & $\begin{array}{c}\mathrm{K}_{\mathrm{w}} \\
\times 10^{-3} \\
\left(\mathrm{~h}^{-1}\right)\end{array}$ & $\begin{array}{c}\mathrm{K}_{\mathrm{s}} \\
\times 10^{-3} \\
\left(\mathrm{~h}^{-1}\right)\end{array}$ & $\begin{array}{c}\mathrm{M}_{\infty} \\
(\mathrm{Kg} / \mathrm{Kg})\end{array}$ & $\begin{array}{c}\mathrm{S}_{\infty} \\
(\mathrm{Kg} / \mathrm{Kg})\end{array}$ \\
\hline 55 & 35 & 3.328 & 0.717 & 1.367 & 4.455 \\
\hline 65 & 35 & 14.92 & 0.721 & 0.601 & 7.858 \\
\hline 75 & 35 & 26.98 & 1.381 & 0.508 & 2.868 \\
\hline 55 & 45 & 8.230 & 0.294 & 0.873 & 19.857 \\
\hline 65 & 45 & 4.694 & 1.114 & 1.265 & 4.859 \\
\hline 75 & 45 & 2.054 & 1.078 & 2.083 & 2.598 \\
\hline
\end{tabular}




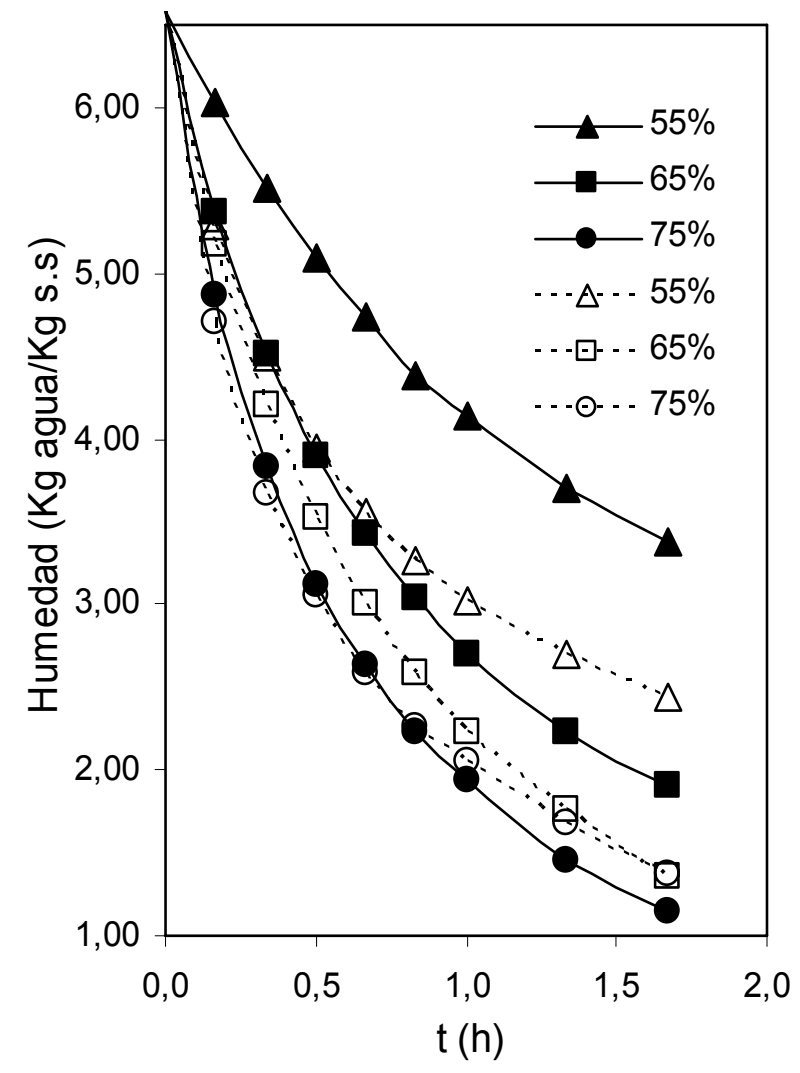

Fig. 1: Variación del contenido de humedad a $35^{\circ} \mathrm{C}\left(\right.$ ) y $45^{\circ} \mathrm{C}($ )..

Puede notarse que a la concentración de $55 \%$ de sacarosa y $35^{\circ} \mathrm{C}$, la diferencia de concentraciones tiene mayor influencia en la transferencia de agua, debido quizá a la menor viscosidad de la solución osmótica que impregna masivamente al producto, resultando en una ganancia de agua, debido a la dificultad de los solutos para penetrar en la fruta. Conforme aumenta la concentración de sacarosa, se puede observar que aumenta la presión osmótica de la solución y con esto la pérdida de agua, es decir, prevalece la deshidratación, aunque la velocidad de transferencia de sólidos sigue siendo menor.

Para $45^{\circ} \mathrm{C}$, la velocidad de transferencia de agua disminuye conforme aumenta la concentración. Dado el aumento en la viscosidad de la solución, no se favorece su salida de la fruta. Esto puede corroborarse también por los valores en equilibrio, donde el menor contenido de humedad se obtiene a la menor concentración, así como la mayor ganancia de sólidos. Por otro lado, a pesar de que la aplicación de vacío contribuye a la apertura de la estructura del higo y la penetración de sólidos, los cuales llegan a acumularse en los espacios intercelulares, puede llegarse a una concentración tal que se presente la satura- ción de la misma, bajando la velocidad de transferencia.

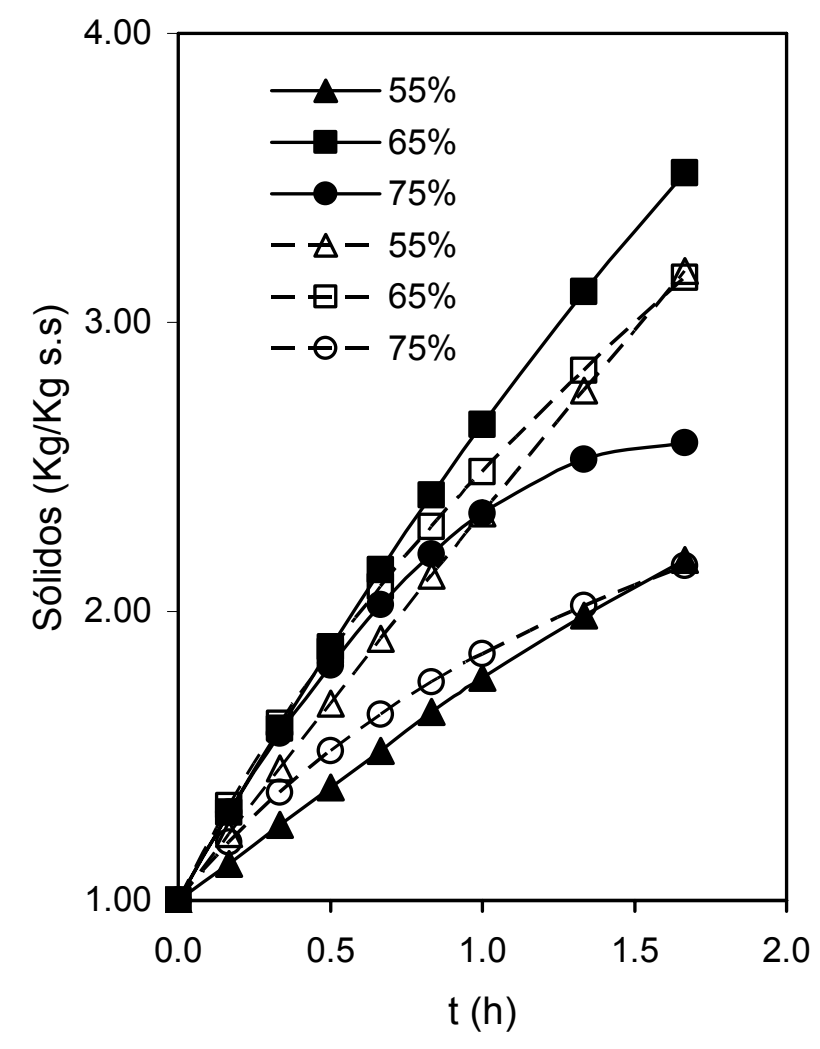

Fig. 2: Variación del contenido de sólidos a $35^{\circ} \mathrm{C}(-)$ y $45^{\circ} \mathrm{C}(-\cdot)$.

En la tabla 2 se presentan los coeficientes de difusión para agua y sólidos $\left(\mathrm{De}_{\mathrm{w}}\right.$ y $\left.\mathrm{De}_{\mathrm{s}}\right)$, calculados a partir de la linealización de las ecuaciones 3 y 4 . Se pueden observar los mismos efectos de la temperatura y la concentración de sacarosa durante el proceso de deshidratación osmótica, de manera que los valores mayores, para ambos coeficientes, se tienen en una concentración de $75 \%$ de sacarosa y $35^{\circ} \mathrm{C}$, en donde la diferencia en la presión osmótica, la viscosidad de la solución y la presión de vacío se combinan de tal manera que permiten la mejor deshidratación del higo.

Tabla 2: Coeficientes de difusión de agua y sólidos para higos

\begin{tabular}{|c|c|c|c|}
\hline $\begin{array}{c}\text { Conc. } \\
(\%)\end{array}$ & $\begin{array}{c}\text { Temp. } \\
\left({ }^{\circ} \mathrm{C}\right)\end{array}$ & $\begin{array}{c}\mathrm{De}_{\mathrm{w}} \times 10^{-6} \\
\left(\mathrm{~m}^{2} / \mathrm{s}\right)\end{array}$ & $\begin{array}{c}\mathrm{De}_{\mathrm{s}} \times 10^{-6} \\
\left(\mathrm{~m}^{2} / \mathrm{s}\right)\end{array}$ \\
\hline 55 & 35 & 0.585 & 0.126 \\
\hline 65 & 35 & 2.624 & 0.127 \\
\hline 75 & 35 & 4.744 & 0.242 \\
\hline 55 & 45 & 1.447 & 0.051 \\
\hline 65 & 45 & 0.825 & 0.196 \\
\hline 75 & 45 & 0.361 & 0.189 \\
\hline
\end{tabular}

Información Tecnológica - Vol. 18 Nº 2 - 2007 
Con el coeficiente de difusión se puede observar la menor rapidez en el transporte de solutos a $55 \%$ de sólidos en solución y $45^{\circ} \mathrm{C}$, donde la diferencia de concentraciones es la principal fuerza motriz de la transferencia. $Y$ al aumentar la concentración, la presión osmótica favorece la difusión del agua hacia la solución, por lo que se tienen mayores coeficientes efectivos de agua que de solutos.

Por otra parte varios autores coinciden en que las comparaciones de difusividad del agua como de sólidos durante el proceso de deshidratación son difíciles debido a los diferentes métodos y modelos empleados para estimar la difusividad, pero lo mas importante son las diferencias en la variación, en la composición y sobre todo en la estructura de los diferentes alimentos empleados. (Cardoso,2007; Singh, 2006).

\section{CONCLUSIONES}

La influencia de la concentración y la temperatura de la solución osmótica en el proceso de deshidratación osmótica son muy importantes, aunque se puede decir que la variación en los valores de los coeficientes dependen también de los cambios en las propiedades del alimento por la aplicación del vacío y el cambio en temperaturas (Kaymak y Sultanoglu, 2000).

La presión de vacío redujo el tiempo de proceso en la deshidratación osmótica y se obtuvieron coeficientes de difusión mayores en comparación con otros productos deshidratados osmóticamente, así como para los higos deshidratados por aire caliente (Rastogi y Raghavarao, 2004; Babalis y Belessiotis, 2004).

Aunque en general el aumento en la concentración de la solución y temperatura favorece la transferencia de masa, el cambio en las características estructurales del producto bajo proceso son determinantes para las velocidades de deshidratación que puedan obtenerse.

\section{NOMENCLATURA}

$D e_{w} \quad$ Coeficiente de difusión efectivo para la humedad $\left(\mathrm{m}^{2} / \mathrm{s}\right)$

$D e_{s} \quad$ Coeficiente de difusión efectivo para los sólidos $\left(\mathrm{m}^{2} / \mathrm{s}\right)$

\footnotetext{
$K_{w} \quad$ Coeficiente de transferencia de masa para la humedad $\left(h^{-1}\right)$

$K_{s} \quad$ Coeficiente de transferencia de masa para los sólidos $\left(h^{-1}\right)$

$r \quad$ Radio del higo $(m)$

Mo , $M_{t}$ Concentración de humedad inicial, al

$M_{\infty} \quad$ tiempo t y en el equilibrio ( $\mathrm{kg}$ agua $/ \mathrm{kg}$ sólido seco)

$S_{0} S_{t} \quad$ Concentración de sólidos inicial, al

$S_{\infty} \quad$ tiempo $\mathrm{t}$ y en el equilibrio $(\mathrm{kg} / \mathrm{kg}$ sólido seco)

$t, t_{0}, t_{\infty}$, Tiempo, tiempo inicial y tiempo en el equilibrio $(s)$

$-d m / d t \quad$ Variación del contenido de humedad con respecto al tiempo

$d s / d t \quad$ Variación del contenido de sólidos con respecto al tiempo

MR Proporción de humedad

MS Proporción de solutos
}

\section{REFERENCIAS}

AOAC Official Method of Analysis. 15 $5^{\mathrm{a}}$ ed., Association of Official Analytical Chemists. Washington, DC (1990).

Babalis, S.J. y V.G. Belessiotis, Influence of the drying conditions of the drying constants and moisture diffusivity during the thin-layer drying of figs, Journal of Food Engineering: $65,449-458$ (2004).

Barbosa-Canovas, G., Deshidratación de alimentos. Acribia, España (2000).

Cardoso, A.S. A., Barros, N. B., Cavalcanti, N. A., Moreira, A. P; Barbosa, G. N, Evaluation of water and sucrose diffusion coefficients during osmotic dehydration of jenipapo (Genipa Americana L.) Journal of Food Engineering: 78, 551-555 (2007).

Chenlo, F., Moreira, C., Fernandez-Herrero, C., Vazquez, G, Mass transfer during osmotic dehydration of chesnut using sodium chloride solutions, Journal of Food Engineering: 73, 164-173 (2006).

Crank, J., The Mathematics of Diffusion. Oxford Science Press Inc., N.Y. EUA (1975).

Gómez, A. D., Lucena, B. J., Colato, A. G., Xidieh. M. F. E., Osmotic dehydration of acerola fruit (Malpighia punicifolia L.) Journal of Food Engineering: 68, 99-103 (2005). 
Jongen, W., Fruit and vegetable processing: Improving quality. Woodhead Publishing Limited. England (2002).

Kaymak, F. y M. Sultanoglu, Modelling of mass transfer during osmotic dehydration of apple, Journal of Food Engineering: 46, 243247 (2000).

Rastogi, N.K., K.S.M.S Raghavarao, K. Niranjan y D. Knorr, Recent developments in osmotic dehydration: methods to enhance mass transfer, Trends in Food Science \& Technology: 13, 48-59 (2002).

Rastogi N.K. y K.S.M.S. Raghavarao, Mass Transfer during osmotic dehydration of pineapple: Considering Fickian diffusion in cubical configuration, Lebensm.-Wissu. Tech: 37, 4347 (2004).
Sánchez, Ma. Teresa., Proceso de conservación poscosecha de productos vegetales. A. Madrid Vicente, Ediciones. España (2004).

Sharma y S. Kamal (editores), Ingeniería de Alimentos: Operaciones Unitarias y Prácticas de Laboratorio, Limusa, México (2003).

Singh, B., Kumar, A., Gupta, A.K. Study of mass transfer kinetics and effective diffusivity during osmotic dehydration of carrot cubes. Journal of Food Engineering: Article in press (2006).

Pan, Y.K., L.J. Zhao, Y. Zhang, G. Chen, y A.S. Mujumdar, Osmotic Dehydration Pretreatment in Drying of Fruits and Vegetables, Drying Technology : 21(6), 1101-1114 (2003). 